\title{
The ever-evolving role of pathologists in the management of breast cancer with neoadjuvant treatment: recommendations based on the Spanish clinical experience
}

\author{
O. Burgués ${ }^{1}\left(\mathbb{D} \cdot M^{\mathrm{a}}\right.$. Á. López-García ${ }^{2} \cdot$ B. Pérez-Míes ${ }^{3} \cdot$ P. Santiago $^{4} \cdot$

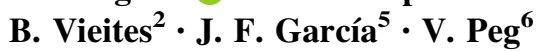

Received: 30 May 2017/ Accepted: 25 July 2017/Published online: 9 August 2017

(C) The Author(s) 2017. This article is an open access publication

\begin{abstract}
Purpose To compare the current international standards for neoadjuvant systemic therapy (NAST) protocols, and establish consensus recommendations by Spanish breast pathologists; and to look into the Spanish reality of defining pathological complete response in daily practice.

Materials and methods A modified Delphi technique was used to gain consensus among a panel of 46 experts with regard to important issues about NAST specimens, with the objective of standardize handling and analysis of these breast cancer specimens. In addition, a survey was conducted among 174 pathologists to explore the Spanish reality of post-NAST breast cancer specimens handling. Results Our survey shows that pathologists in Spain follow the same guidelines as their international colleagues and face the same problems and controversies. Among the experts, $94.1 \%$ agreed on the recommendation for a pretreatment evaluation with a core needle biopsy, and $100 \%$ of experts agreed on the need of having properly indicated
\end{abstract}

O. Burgués

burgues_oct@gva.es

1 Servicio de Anatomía Patológica, Hospital Clínico Universitario, Avda. Blasco Ibáñez, 17, 46010 Valencia, Spain

2 Servicio de Anatomía Patológica, Hospital Virgen Del Rocío, Seville, Spain

3 Servicio de Anatomía Patológica, Hospital Universitario Ramón y Cajal, Madrid, Spain

4 Servicio de Anatomía Patológica, Complejo Hospitalario A Coruña, A Coruña, Spain

5 Roche Farma S.A., Madrid, Spain

6 Servicio de Anatomía Patológica, Hospital Vall d'Hebron, Barcelona, Spain information for the post-NAST surgical specimens. However, only $82.7 \%$ of them receive properly labelled specimens and even less receive specimens where markers are identified and the degree of clinical/radiological response is mentioned. Among participants $59.9 \%$ were familiar with the residual cancer burden system for post-NAST response quantification, but only $16.1 \%$ used it regularly.

Conclusions Active participation on breast cancer multidisciplinary teams, optimal usage of core needle biopsy for timely and standardized procedures for the diagnostic analysis, and accurate diagnosis of pathological complete response and complete evaluation of the response to NAST need to become the standard practice when handling breast cancer specimens in Spain.

Keywords Breast cancer - Neoadjuvant treatment . Pathology $\cdot$ Recommendation

\section{Introduction}

Detailed and accurate histo-pathological analysis and description of cancer specimens are pivotal to any decisions taken regarding treatment options. In clinical oncology practice, planning of local or systemic treatment either pre-operatively or post-operatively is based on predictive and prognostic information extracted from histo-pathological and immunohistochemical data. Evidence-based treatment protocols tailored to each cancer characteristics can be elaborated by an in-depth understanding of the tumour's distinct pathologic features.

It has been shown that structured pathology reports with a standardized format significantly improve the data quality and completeness $[1,2]$ contributing thus importantly to the management of oncologic patients and simplifying the 
auditing process at the same time. Standardized pathology reports have been implemented via publication of national guidelines and protocols for histo-pathological assessments of breast cancer specimens in USA [3], UK [4], Australasia [5], Belgium [6], The Netherlands [7] and Germany [8].

With the increasingly better understanding of breast cancer and the emergence of new treatments, the management of this disease becomes progressively adjusted to the patient's profile and tumour behaviour. A multidisciplinary assessment process combining pre-operative clinical findings with imaging results, percutaneous biopsy and surgical histo-pathological analysis is of fundamental importance in the delivery of a high quality care for the breast cancer patients.

Currently, an increasing number of early-stage breast cancer patients are treated with neoadjuvant systemic therapy (NAST) protocols with the aim to increase the possibilities of breast conservation, eliminate micrometastases and also to measure efficacy of systemic therapy in vivo as assessed by means of pathologic response. Pathologic response after NAST is a significant prognostic indicator [9]; patients with a pathological complete response (pCR) have the best prognosis.

When we talk about NAST it does not necessarily mean chemotherapy schemes; it could equally be an anti-oestrogen treatment in combination with a biologic blocking agent or different combinations with anti-HER2 agents. In most institutions the decisions regarding NAST follow a multidisciplinary process and universally established criteria.

However, NAST might have an important impact for pathologists as handling and reporting of breast cancer specimens after NAST require specific considerations. Because of the complexity of NAST specimens, a multidisciplinary approach is essential before, during and after NAST. An accurate localization and sampling of the tumour bed is necessary for a complete evaluation of the original tumour. That makes gross pathologic methods the most important source for accurate definition of pCR [10] and emphasizes the need for a universally standardized protocol to evaluate the post-NAST specimen.

Multicenter breast cancer clinical trial studies have indicated that there is a huge variation in handling and reporting these specimens [11], due to the fact that guidelines are not always followed accurately [12]. Currently there are different classification systems with several relevant reviews [13-16], where recommendations for the assessment of pathologic response to NAST can be found. In recent time, NAST has been part of multicentre clinical trials and often recommendations for assessment of pathology specimens are elaborated during these trials $[17,18]$.
The pathologist's task in assessing post-NAST specimens could get even more complicated by the fact that the definition of pCR is not uniformly accepted creating thus further challenging issues with the interpretation and reporting of the data [19]. Bossuyt et al. and Provenzano et al. in their respective studies $[17,18]$ recommended that the post-NAST assessment and report should include information on $\mathrm{pCR}$ versus residual disease as defined by the FDA meta-analysis [9] (ypTO/is ypNO), AJCC/UICC $y p T$ plus $y p N$ stage and detailed quantification of residual disease (RCB system).

In this ever-changing era in the diagnosis and treatment of breast cancer, pathologists, the keepers and purveyors of excised tissues, have extended their role by employing new technologies to examine the molecular complexities of breast cancer relating thus its biological heterogeneity to prognosis and treatment, and issuing recommendations aiming to improve the handling and reporting of the specimens [20]. With this paper we aspire to contribute positively to this global effort of post-NAST breast cancer specimens reporting standardization by looking into the Spanish reality of daily practice of accurately defining pCR. We compare it with the current international standards and establish relevant recommendations elaborated by Spanish breast pathologists reaching consensus following a Delphi methodology enquiry with the aim to improve management and prognosis of this disease.

\section{Materials and methods}

\section{The Delphi method}

A modified Delphi technique was used to gain consensus among a panel of experts [21]. The Delphi method is a structured process used to gain consensus on a given question from a panel (Delphi panel) constituted by a range of experts. With this methodology, situations such as dominance of the panel by the views of few can be avoided [22].

A previously elaborated questionnaire containing assertions covering current controversial issues on the management of breast cancer patients with NAST in the daily Spanish practice and on handling and evaluation of post-NAST breast cancer samples was presented to a group of pathologists with special interest in breast cancer participating at the time in an interactive session to establish the role of the pathologist in the management of breast cancer with NAST. Each member of the group expressed their opinion to each assertion by voting anonymously. The degree of the participants' agreement or disagreement with the questionnaire's assertions was scored. 
The responses' results from this first round of voting were subjected to statistical analysis to assess the degree of consensus or not for each assertion among the participants and subsequently were presented for discussion to the entire group giving thus the opportunity to each group member to reconsider their responses. In a second round of voting, ambiguous items with relevant proposals by comments of the first round were subjected to a further survey. The results from this second round were analysed once again to determine the items that reached sufficient degree of consensus among the participating experts, and which ones could be used to issue relevant recommendations.

\section{Stages of the process}

Following an extensive review of literature on the subject of pathological evaluation of post-NAST specimens of breast cancer, taking in account the experience from the Spanish clinical practice and paying special attention to the recently published recommendations of Provezano et al. [17] and Bossuyt et al. [18], two questionnaires, one containing assertions on relevant items of handling and analysing post-NAST breast cancer samples (consensus questionnaire) and the other questions about the Spanish daily breast cancer pathology practice (Clinical practice questionnaire) were designed by a committee of pathology experts on breast cancer to be discussed and subjected to consensual surveyance by the Delphi method.

Clinical practice questionnaire was presented to a group of pathologists who were participating in a training meeting that included the Delphi method questionnaire (see below) and to 126 pathologists participating in training sessions throughout Spain (Valencia, Madrid, A Coruña, and Sevilla). In total, 174 pathologists answered the clinical practice questionnaire.

Consensus questionnaire was presented to a group of pathologists (30 specialists in breast cancer, 16 general pathologists), who were participating at the time in an interactive session regarding the role of the pathologist in the management of breast cancer treated with NAST. The items of the consensus questionnaire were scored by means of a Likert-type ordinal scale from 1 to 9 points (minimum 1: complete disagreement, maximum 9: complete agreement) as it is described by the UCLA-RAND Corporation [21]. The responses were classified into three groups of answers (1-3: disagree, 4-6: neither agree nor disagree and 7-9: agree). An item was regarded as consensual in agreement if the median of the scores fell within the $\{7-9\}$ range group. On the contrary, it was considered consensual in disagreement if the median fell within the $\{1-3\}$ range group. Items with medians within the $\{4-6\}$ range group should be subjected to a second round of voting. The questions of the clinical practice questionnaire were subjected to general voting.

The answers to the questionnaires were analysed by external reviewers. Although consensus was reached for all items of the consensus questionnaire during the first round of voting, items that reached consensus between 70 and $80 \%$ were subjected to a second round of voting after presenting and discussing the results of the first round of voting. At the end of this round consensus was reached for all items. The final results were presented to the participants for further discussion and comments, which were used to compose the recommendations document by a scientific committee, whose members reviewed and approved the final draft unanimously.

\section{Results}

Consensus was defined a priori as greater than $70 \%$ agreement on all items with the same ranking according to the recommended quality indicators for a Delphi study [23]. The consensus questionnaire contained 18 items addressing 8 current and controversial issues in the handling and analysis of the post-NAST breast cancer pathology specimens (Table 1). As it has been mentioned already consensus was reached for all of the items during the two rounds of voting (100\%), and the detailed results can be seen in Table 1. During the ensuing discussion at the end of the voting there was general agreement by all the participants regarding the results of the voting and no concerns were expressed with regards to the consensus reached.

The results/recommendations of the consensus questionnaire, which are very much in line with the current international recommendations, are summarized below:

1. For the initial evaluation of treatment a core needle biopsy (CNB) is recommended and an unequivocal diagnosis of infiltrating breast cancer with histological type, grade (Nottingham/Scarff-Bloom) and biomarkers [oestrogen receptor (ER), progesterone receptor (PR), HER2, Ki-67] should be included. The tumour should be marked during the biopsy.

2. For the evaluation of the axilla during the diagnostic process it is recommended: axillary ultrasound (US) for all those patients that undergo CNB for suspected breast cancer, with a fine needle aspiration biopsy (FNA) or a CNB in the case of suspicious lymph nodes detection. It is recommended the evaluation of sentinel lymph node (SLN) biopsy after treatment, including positive lymph nodes at the moment of diagnosis, which become negative post-NAST. In the case of 
Table 1 Consensus questionnaire (with Delphi results)

\begin{tabular}{|c|c|c|}
\hline Pre-treatment diagnosis & Agreement $(\%)$ & Result \% \\
\hline $\begin{array}{l}\text { For the pre-treatment evaluation a core needle biopsy }(\mathbf{C N B}) \text { is } \\
\text { recommended }\end{array}$ & 94.1 & First round agreement \\
\hline $\begin{array}{l}\text { The pre-treatment evaluation should include } \\
\text { Unequivocal diagnosis of invasive cancer } \\
\text { Histological type (OMS 2012) } \\
\text { Histological grade (Nottingham Scarff-Bloom) } \\
\text { Biomarkers: ER-PR-HER } 2 \text {-Ki67 }\end{array}$ & 100 & First round agreement \\
\hline $\begin{array}{l}\text { During the biopsy a marking clip should be left } \\
\text { Axillary evaluation at diagnosis }\end{array}$ & 94.4 & First round agreement \\
\hline $\begin{array}{l}\text { It is recommended } \\
\text { US axillary evaluation for all the breast cancer suspected patients who } \\
\text { undergo CNB } \\
\text { Perform FNA or CNB for morphologically abnormal nodes }\end{array}$ & 97.2 & First round agreement \\
\hline $\begin{array}{l}\text { Sentinel lymph node biopsy (SLNB) is recommended post-treatment } \\
\text { including positive nodes at diagnosis } \\
\text { Required information on the request form for post-NAST surgical specimen }\end{array}$ & 79.3 & Second round agreement \\
\hline The request form should indicate clearly that NAST was done & 97.3 & First round agreement \\
\hline $\begin{array}{l}\text { The request form should include information regarding site, number and size } \\
\text { of the tumour/s pre-treatment }\end{array}$ & 100 & First round agreement \\
\hline $\begin{array}{l}\text { The following information should be indicated } \\
\text { Presence and type of markers (clips, tattoos etc.) } \\
\text { Pre-treatment nodal status } \\
\text { Clinical/radiological grade of response (breast and axilla) } \\
\text { Characteristics of the primary tumour (type, HG, ER, PR, } \mathrm{HER}_{2}, \mathrm{Ki} 67 \text { ) } \\
\text { Macroscopic analysis post-NAST }\end{array}$ & 100 & First round agreement \\
\hline $\begin{array}{l}\text { A systematic sampling of the specimen and the creation of schematic map, } \\
\text { using imaging or individual drawings. }\end{array}$ & 100 & First round agreement \\
\hline $\begin{array}{l}\text { If the tumour/tumour bed are detectable macroscopically their presence and } \\
\text { size should be indicated }\end{array}$ & 100 & First round agreement \\
\hline $\begin{array}{l}\text { The sampling should include both macroscopic lesions and the suspected } \\
\text { areas affected by the tumour pre-treatment }\end{array}$ & 100 & First round agreement \\
\hline $\begin{array}{l}\text { All lymph nodes should be evaluated histologically and they should be } \\
\text { included in } 1-2 \mathrm{~mm} \text { slices }\end{array}$ & 96.4 & Second round agreement \\
\hline $\begin{array}{l}\text { The post-NAST histology report should include } \\
\text { Grade and histological type: Multiple tumours with different histological } \\
\text { type or grade and clearly separated by adipose tissue should be reported } \\
\text { separately }\end{array}$ & 100 & First round agreement \\
\hline $\begin{array}{l}\text { Size and extension: The following two options are recommended for } \\
\text { inclusion in the report; two dimensions of the tumour area including not } \\
\text { only the dispersed residual tumour foci but also the tumour bed among all } \\
\text { of them, or according to the AJCC ( } 7 \text { th ed) the size }(y p T) \text { is established } \\
\text { based on the biggest adjacent invaded area. It should be indicated that the } \\
\text { tumour is multifocal }\end{array}$ & & \\
\hline $\begin{array}{l}\text { Cellularity: If the evaluation system is based on cellularity reduction } \\
\text { (Miller-Payne, Pinder), then it should be compared with the CNB at } \\
\text { diagnosis }\end{array}$ & & \\
\hline Tumour bed: detectable or not, and its position regarding the margins & & \\
\hline Lymphovascular invasion: its presence excludes pCR & & \\
\hline Nodal metastasis: the presence of cancer cells in lymph nodes is not $\mathrm{pCR}$ & & \\
\hline $\begin{array}{l}\text { Treatment response: presence or absence of treatment response and number } \\
\text { of nodes with regressive changes }\end{array}$ & & \\
\hline
\end{tabular}


Table 1 continued

\begin{tabular}{|c|c|c|}
\hline Pre-treatment diagnosis & Agreement $(\%)$ & Result \% \\
\hline \multicolumn{3}{|l|}{ Post-NAST sentinel node selective biopsy (SLNB) } \\
\hline $\begin{array}{l}\text { For the evaluation of sentinel node post -NAST techniques that allow for } \\
\text { treatment response assessment should be used }\end{array}$ & 81.5 & Second round agreement \\
\hline \multicolumn{3}{|l|}{ Post-NAST biomarkers evaluation } \\
\hline $\begin{array}{l}\text { Repeated evaluation of biomarkers post-NAST in residual invasive cancer } \\
\text { specimens (breast or nodes) should be reserved for cases that }\end{array}$ & 100 & Second round agreement \\
\hline \multicolumn{3}{|l|}{ The pre-NAST result was negative } \\
\hline \multicolumn{3}{|l|}{ The pre-NAST result was inconclusive } \\
\hline \multicolumn{3}{|l|}{ In case of tumours without any response to treatment } \\
\hline \multicolumn{3}{|l|}{$\begin{array}{l}\text { In case of heterogeneous tumours or multifocal ones with different } \\
\text { morphology }\end{array}$} \\
\hline \multicolumn{3}{|l|}{ Pathologic complete response ( $p C R$ ) } \\
\hline $\begin{array}{l}\text { pCR is defined by the absence of infiltrating residual cancer not only in the } \\
\text { totally excised breast specimen but also in all sampled regional nodes post- } \\
\text { NAST completion }\end{array}$ & 96.7 & First round agreement \\
\hline The presence of carcinoma in situ only is considered to be pCR & 93.8 & First round agreement \\
\hline \multicolumn{3}{|l|}{ Response valuation system } \\
\hline $\begin{array}{l}\text { For the post-NAST response evaluation the RCB (residual cancer burden) } \\
\text { system from MDACC (Symmans) is recommended }\end{array}$ & 92.9 & Second round agreement \\
\hline
\end{tabular}

positive lymph node detection in the axilla, axillary lymphadenectomy is recommended.

3. The requesting form for the histological evaluation of post-NAST specimens should clearly indicate that the specimen is a post-NAST one. The pathologist must have information regarding the number and size of the tumour/s before treatment, the existence/types of markers, nodal status before treatment, characteristics of the primary tumour (type, histological grade (HG), ER, PR, HER2, Ki67) and the clinical/radiological response grade in both breast and axilla after treatment.

4. With regards to the macroscopic examination of the post-NAST specimen, the recommendations consist of systematic sampling and mapping of the specimen using a photograph, X-ray or a draw sketch of the slices (the sampling should be extended to include the entire suspected tumoural area before the treatment as well), localization and measurements of the tumour/tumour bed (if and when they can be identified) and histological evaluation of all included lymph nodes (in 1-2 mm slices) (Fig. 1).

5. The pathology report should include the following information: histological type and grade (in the case of multiple tumours with different types/grades a separate report for each one of them should be issued), size and extension of the neoplasm (either a two-dimensional calculation in $\mathrm{mm}$ of the neoplastic area including not only the dispersed tumour infiltrated residual areas but the tumoural bed between them as well known as residual cancer burden RCB estimation [24], or according to the AJCC [25] the size $y p T$ of a multifocal tumour is established based on the biggest adjoining invaded area), the percentage of tumour cellularity, margin evaluation (including invasion by the tumour bed), lymphovascular invasion, number of lymph nodes showing metastases indicating the size of the biggest one and finally the presence or not of posttreatment changes in the lymph nodes.

6. Biomarkers in residual invasive breast cancer specimens (breast or nodes) post-NAST should be assessed in cases where the results before the treatment were not conclusive or were negative, in tumours with minimal response to treatment and in heterogeneous tumours or in multifocal cancers with different morphologies.

7. Post-NAST pCR is defined by the absence of residual invasive cancer not only in the totally resected surgical specimen but also in all of the sampled lymph nodes following the completion of NAST as well. Lymphovascular invasion and/or isolated tumour cells (ITC) in the nodes are not considered as pCR. The presence of in situ carcinoma solely is classified as pCR. The use of the RCB system is recommended for the evaluation of the post-NAST breast cancer response [24].

The analysis of the results of the clinical practice questionnaire is summarized on Table 2. It is worth noting the percentage of the panellists that regularly participate in the multidisciplinary breast cancer meeting, which was around $70 \%$. Almost all of the participants $(97.9 \%)$ responded that the axilla is evaluated during the diagnostic 


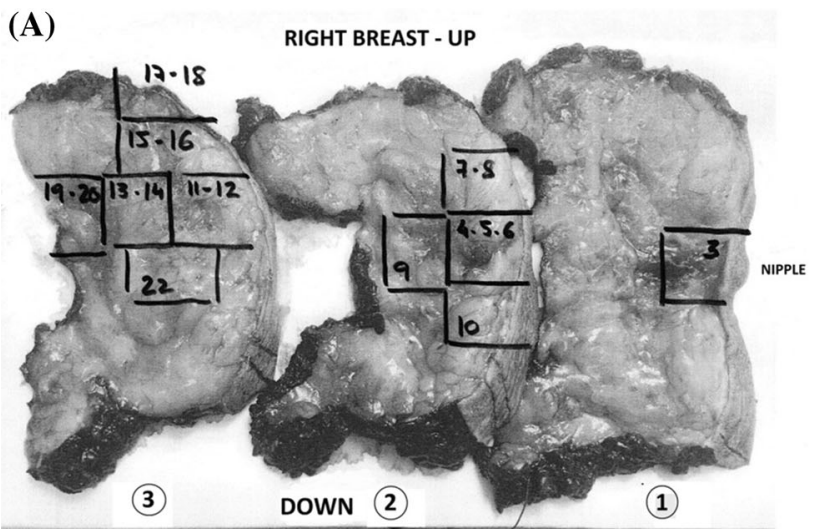

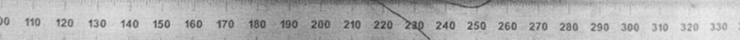

(B)

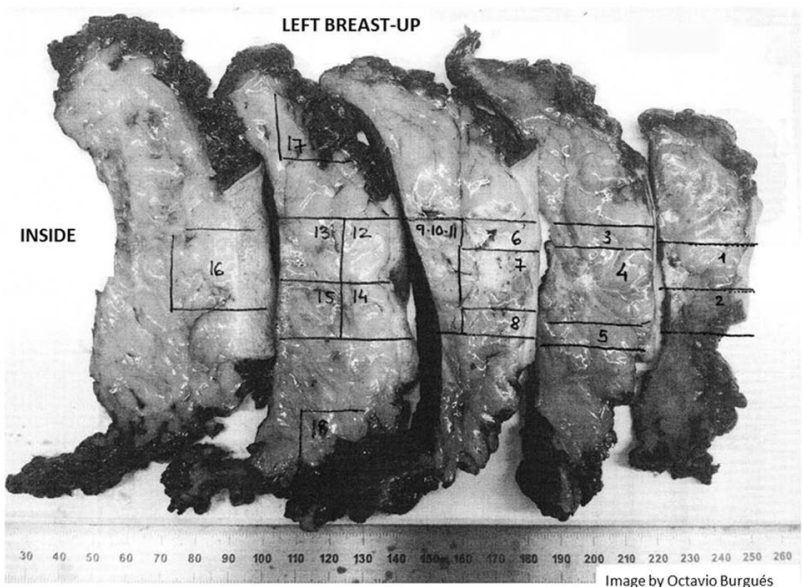

Fig. 1 Mastectomy specimens in which a complete mapping of the tumour bed or suspected areas with residual tumour has been carried out to assess pathological response to the neoadjuvant treatment. Alternatively to specimen photographs, X-rays or diagrams may be used

process and FNA or CNB is performed for cancer suspicious nodes. Regarding the sentinel lymph node $44.8 \%$ thought that a biopsy should be done pre-NAST, 30.7\% post-NAST and only to patients with negative nodes at diagnosis, $17.5 \%$ post-NAST, including patients with positive nodes at diagnosis as well, $5.6 \%$ pre-and postNAST and $2.1 \%$ said that there is no need for selective sentinel lymph node biopsy. $42.6 \%$ use the OSNA method intra-operatively to evaluate the sentinel lymph node, $24.1 \%$ imprint cytology or frozen section intra-operatively and $33.3 \%$ evaluate the lymph nodes at later stage (not intra-operatively).

Among the participants, $59.9 \%$ were familiar with the RCB system [24, 26] for the quantification of the postNAST response, but this system was used as the method of choice only by $16.1 \%$ of them, whereas $62.7 \%$ were using the Miller-Payne classification system [27]. $16.1 \%$ were using both systems. With regards to the definition of the
pCR, 59.1\% responded non-invasive cancer and ypTO/ ypTis ypNO.

\section{Discussion}

The last few years NAST is offered more and more often to patients with early breast cancer and it has almost become routine for patients to receive systemic therapy [11, 28]. However as a result of NAST, the correlation of clinicopathological and radiological response with residual tumour could not be defined accurately [29], although with the introduction of magnetic resonance the accuracy in discriminating residual disease versus pCR has improved [30]. Pathologic evaluation of the tumour is still regarded as the gold standard in optimizing the knowledge required for the design of any breast cancer therapy. Pathologists have an important role in providing this information, standardizing existing classification schemes and developing new schemes for ongoing trials [15].

Post-NAST breast cancer specimens reporting standardization recommendations for clinical trials are key for accurate data collection during these studies; however, we believe that these recommendations should be applied to any post-NAST breast cancer specimens in routine clinical practice in an effort to standardize pathological response reporting.

Every health carer that participates in a multidisciplinary team for neoadjuvant-treated breast cancer patients has to deal with critical issues and the pathologist is not an exception to this concept. Handling of surgical breast cancer specimens can be challenging, because of a number of histo-pathological changes in the post-NAST tumour and adjacent breast tissues [31]. However, only minimal guidelines regarding specimen handling do exist [32]. Frequently, pathologists in their daily routine practice have to deal with specimens where important parameters for reporting are missing. The neo-tAnGo trial revealed large variations in handling and reporting of post-NAST breast cancer specimens [11].

The results of the clinical practice questionnaire showed similar results; only $70 \%$ of the participants attend the breast cancer MDT, $82.7 \%$ of them receive properly labelled specimens and even less receive specimens where markers are identified and the degree of clinical/radiological response is mentioned, whereas with the Delphi process there was consensus that the histological evaluation requesting form should contain information such as whether or not the specimen is a post-NAST one, number and size of the tumour/s before treatment, the existence/types of markers, nodal status before treatment, characteristics of the primary tumour (type, HG, ER, PR, HER2, Ki67) and 
Table 2 Clinical practice questionnaire. The total number of participants was 174 , not all participants answered all questions

\begin{tabular}{|c|c|c|}
\hline Question & Responses $(\%)$ & $N$ \\
\hline $\begin{array}{l}\text { Participation in multidisciplinary breast cancer meeting by the participant or } \\
\text { any other pathologist from the team (yes) }\end{array}$ & 70.7 & 174 \\
\hline \multicolumn{3}{|l|}{ Regarding the information provided in post-NAST surgical specimens } \\
\hline Specimens are properly marked as post-NAST (yes) & 82.7 & 168 \\
\hline \multicolumn{3}{|l|}{ Information accompanying the post-NAST surgical specimens } \\
\hline Tumour location and size before treatment (yes) & 78.8 & 170 \\
\hline Radiological clips are identified (yes) & 74.3 & 167 \\
\hline Degree of clinical and radiological response (yes) & 64.9 & 168 \\
\hline $\begin{array}{l}\text { Information regarding about tumour features before treatment (type and } \\
\text { histological grade, ER, PR, HER2, Ki-67) (yes) }\end{array}$ & 88.4 & 172 \\
\hline \multicolumn{3}{|l|}{ Regarding the evaluation of the axilla and sentinel lymph node } \\
\hline $\begin{array}{l}\text { Routine evaluation of axilla at the time of diagnosis (with sentinel lymph } \\
\text { node biopsy in uncertain cases) }\end{array}$ & 97.6 & 163 \\
\hline Routine sentinel lymph node biopsy in NAST patients & & 143 \\
\hline Pre-NAST & 44.8 & \\
\hline Post-NAST only in patients with negative lymph nodes at diagnosis & 30.7 & \\
\hline Post-NAST for all patients regardless of lymph node status at diagnosis & 17.5 & \\
\hline Pre- and Post-NAST & 5.6 & \\
\hline Not done & 2.1 & \\
\hline Method used to assess sentinel lymph node & & 162 \\
\hline Imprint cytology or frozen section intra-op & 24.1 & \\
\hline OSNA method & 42.6 & \\
\hline Post-surgery evaluation & 33.3 & \\
\hline Familiar with the RCB system (YES) & 59.9 & 167 \\
\hline \multicolumn{3}{|l|}{ Regarding the evaluation of the specimens } \\
\hline Method used for quantification of the post-NAST response & & 174 \\
\hline Miller-Payne & 62.6 & \\
\hline $\mathrm{RCB}$ & 16.1 & \\
\hline Both & 16.1 & \\
\hline Other & 1.7 & \\
\hline None & 3.5 & \\
\hline Definition of $\mathrm{pCR}$ & & 154 \\
\hline $\begin{array}{l}\text { Absence of residual invasive cancer (with or without DCIS) and negative } \\
\text { lymph node (ypT0/ypTis ypN0) }\end{array}$ & 59.1 & \\
\hline $\begin{array}{l}\text { Absence of residual invasive cancer (with or without DCIS) regardless of } \\
\text { axillar lymph nodes (ypT0/ypTis) }\end{array}$ & 16.9 & \\
\hline $\begin{array}{l}\text { Complete absence of cancer in the breast (no invasive cancer nor DCIS) } \\
\text { and negative lymph nodes (ypT0 N0) }\end{array}$ & 19.5 & \\
\hline $\begin{array}{l}\text { Complete absence of cancer in the breast (no invasive cancer nor DCIS) } \\
\text { regardless of axillar lymph nodes (ypT0/ypTis) }\end{array}$ & 4.6 & \\
\hline Analysis of biomarkers on surgical specimens after TNA & & 158 \\
\hline No, never & 12.0 & \\
\hline Yes, usually & 53.2 & \\
\hline Yes, sometimes & 34.8 & \\
\hline
\end{tabular}

the clinical/radiological response grade (breast, axilla) post-treatment.

Following the Delphi inquiry in the ensuing discussion among the participants, the consensual questionnaire and clinical practice questionnaire results were analysed.
During the pre-treatment assessment a percutaneous image-guided CNB is necessary to determine unequivocally the existence or not of invasion, histological type and grade of the tumour and the relevant biomarkers (ER, PR, HER2, Ki-67) [33] used to evaluate the necessity for NAST 
[34]. Tumour size is usually estimated by clinical examination and radiological tests. At the same time, biopsies could be taken for research purposes [35] and most importantly clips should be placed to mark the site of the tumour which are extremely useful especially in those cases with pCR post-NAST [36].

Axillary status at diagnosis plays an important role in breast cancer patients when decisions regarding local or systemic treatment are taken. The consensus for the evaluation of the axilla during the diagnostic process was that axillary US should be performed to all breast cancer suspected patients that undergo $\mathrm{CNB}$ and in the case of suspicious lymph nodes detection, a biopsy (FNA or/and CNB) of them should be performed [37] with concomitant placement of a radiological marker into the biopsied node. This was in agreement with the current recommendations $[17,18]$ and the results of our consensual and clinical practice questionnaire.

Sentinel lymph node biopsy (SLNB) before NAST is not always indicated because an excision-biopsy of a positive sentinel lymph node might complicate the evaluation of nodal response to NAST and thus it invalidates the yp stage and the calculation of RCB. It should be reserved for cases where the pre-NAST axillary status is required for local or systemic treatment decisions [38]. In our survey the conclusions of the consensus questionnaire with reference to the SLNB were similar, whereas the results of the clinical practice ones were inconclusive (see the results section).

With regards to the handling and macroscopic and microscopic examination of the post-NAST breast cancer specimen the achieved recommendations' consensus (Table 1) was in the same lines with the recently internationally published guidelines [17, 18] taking into account that a post-NAST specimen has special features that are different from a non-NAST specimen [13]. The pathologic evaluation of the post-NAST specimen should be done keeping in mind that adequacy of surgery is achieved, prognostic factors are assessed and provisions were made for the collection of research specimens. Collection of research tissue should be done according to previously published guidelines [35].

The microscopic examination should give information regarding the type and the grade of the tumour, the extent and the cellularity of the tumour, the margins and the existence of lymphovascular invasion. Particular attention should be given to the estimation of cellularity as it can be profoundly affected by NAST. The effect of NAST on tumour cellularity can be heterogeneous. Several scoring systems for grading response are based on comparisons between pre- and post-NAST cellularity [13, 27, 39, 40] and frequently they can not deal with this heterogeneity which can be addressed by the RCB system, which does not use pre-treatment cellularity [26], is easily reproducible
[41], can be satisfactorily used in a prospective manner and contributes to the standardization of macroscopic and microscopic methods. It is worth noting the results of the clinical practice questionnaire with regards to the RCB system; although $59.8 \%$ of the participants were familiar with it, only for $16.1 \%$ was the method of choice in assessing cellularity and $16.1 \%$ were using it in combination with the Miller-Payne system.

Post-NAST axillary nodal status is an important predictor of survival independently of breast response [10, 28, 42-44], however the value of SNLB post-NAST is still under a lot of investigation [38, 45]. Various parameters predict worse survival: the number of affected nodes, the size of the largest metastasis and the existence of micro-metastasis and isolated tumour cells (ITCs). The presence of ITCs $(p N O i+)$ precludes pCR. The current recommendation in clinical trials $[17,18]$ is to use a technique that allows the assessment of treatment response and the measurement of the diameter of the metastases (as this measure is used to calculate RCB). Currently in Spain there are trials to assess the validity of the one step nucleic acid amplification (OSNA, Sysmex, Kobe, Japan) in the assessment of SLN post-NAST.

The issue of testing for biomarkers in residual invasive breast cancer specimen post-NAST remains controversial [46]. In our survey, there was consensus that it should be assessed when the pre-NAST CNB is inconclusive or negative, in non-responding to NAST tumours and in heterogeneous or multifocal ones. Of interest is the result of our clinical practice survey, where $53.2 \%$ stated that biomarkers are checked routinely in the post-NAST specimen. Special attention should be given in the determination of Ki67 expression, which correlates well with longterm outcome [47] and although the analytical reproducibility of Ki67 measurements is of concern [48], it is used frequently for basic risk assessment and is part of some post-NAST multivariate prediction models [49].

Pathologic complete response is defined by the absence of residual invasive cancer not only in the totally resected breast surgical specimen but also in all of the sampled lymph nodes following the completion of NAST as well (the presence of cancer solely in situ is classified as pCR): either ypTO/isypNO or ypTOypNO [50]. However, the significance of residual carcinoma in situ remains controversial as it is shown in various studies $[19,51]$. In our clinical practice questionnaire $76 \%$ responded correctly with regards to the US FDA pCR definition.

As for other consensus approaches, vocal members in the panel could influence decisions; in our study, rating was anonymous and took place before an expert debate. For the clinical questionnaire, results were not only collected from the interactive meeting attended by experienced pathologist, but also from several training sessions; results from 
the interactive meeting and training sessions were similar regarding clinical practice.

In conclusion, without a doubt pathologists are playing a fundamental role in the management of post-NAST breast cancer. As the histological changes of post-NAST breast cancer specimens become even more complex, it is clear that pathologists have a demanding task to standardize handling and analysis of breast cancer specimens to enable the usage of pCR as an indicator to novel therapies. Our survey shows that pathologists in Spain follow the same guidelines with their international colleagues and face the same problems and controversies as well. The way forward is active participation to the breast cancer multidisciplinary teams, optimal usage of CNB for timely and standardized procedures for the diagnostic analysis (type, histological grade and biomarkers) of the breast cancer specimen and finally accurate diagnosis of pCR and complete evaluation of the response to NAST.

Acknowledgements We wish to thank Paul Karagounis, on behalf of Springer Healthcare Communications, for medical writing assistance. This assistance was funded by Roche Farma, Spain.

\section{Compliance with ethical standards}

Conflict of interest J. Francisco García is an employee of Roche Farma Spain, all other authors declare no conflict of interest.

Ethical statement The study has been performed in accordance with the ethical standards of the Declaration of Helsinki and its later amendments. This article does not contain any studies with human participants or animals performed by any of the authors.

Informed consent statement Additional informed consent was obtained from all individual participants for whom identifying information is included in this article.

Open Access This article is distributed under the terms of the Creative Commons Attribution 4.0 International License (http://crea tivecommons.org/licenses/by/4.0/), which permits unrestricted use, distribution, and reproduction in any medium, provided you give appropriate credit to the original author(s) and the source, provide a link to the Creative Commons license, and indicate if changes were made.

\section{References}

1. Mathers M, Shrimankar J, Scott D, Charlton F, Griffith C, Angus B. The use of standard proforma in breast cancer reporting. J Clin Path. 2001;54(10):809-11.

2. Sprigley J, McGowan T, MacLean A, Raby M, Ross J, Kramer S, et al. Standardized synoptic cancer pathology reporting: a population-based approach. J Surg Oncol. 2009;99(8):517-24.

3. College of American Pathologists. Protocol for the examination of specimens from patients with invasive carcinoma of the breast. Cancer protocols and checklists (2012). Available from: www.cap.org. Accessed 26 Feb 2017.

4. NHS Breast Screening Programme (NHSBSP). Pathology reporting of breast disease. Royal College of Pathologists. Datasets and tissue pathways. Available from: www.rcpath.org. Accessed 26 Feb 2017.

5. Royal College of Pathologists of Australasia (RCPA). Royal College of Pathologists of Australasia invasive breast cancer structured reporting protocol (Version 2.0). 2nd ed. Surry Hills: Royal College of Pathologists of Australasia (RCPA); 2012.
6. Lambein K, Van de Vijver K, Faverly D, Colpaert C. Belgian guidelines for laboratory handling and pathology reporting of breast carcinoma after neodjuvant therapy. Belg J Med Oncol. 2011;5:144-53.

7. Integraal Kankercentrum Nederland. Beoordeling na neoadjuvante chemo- of endocriene therapie. Dutch integrated cancer center. Assessment of chemoendocrine therapy (2012). www.oncoline.nl/breastcancer. Accessed 26 Feb 2017.

8. Arbeitsgemeinschaft Gynaekologische Onkologie Studiengruppe (Gynecologic oncology working study group) (AGO). www.ago-online.de

9. Cortazar P, Zhang L, Untch M, Mehta K, Costantino J, Wolmark N, et al Pathological complete response and long-term clinical benefit in breast cancer: the $\mathrm{CTN}_{\mathrm{eo}} \mathrm{BC}$ pooled analysis. Lancet. 2014;384:164-72.

10. Mamounas E, Anderson S, Dignam J, Bear H, Thomas J, Geyer C, et al. Predictors of locoregional recurrence after neoadjuvant chemotherapy: results from combined analysis of National Surgical Adjuvant Breast and Bowel Project B-18 and B-27. J Clin Oncol. 2012;30:3960-6.

11. Provenzano E, Vallier A, Champ R, Walland K, Bowden S, Grier A, et al. A central review of histopathology reports after breast cancer neoadjuvant chemotherapy in the neo-tango trial. Br J Cancer. 2013;108:866-72.

12. Dieras V, Fumolaeu P, Romieu G, Tubiana-Hulin M, Namer M, Mauriac L, et al. Randomized parallel study of doxorubicin plus paclitaxel and doxorubicin plus cyclophosphamide as neoadjuvant treatment of patients with breast cancer. J Clin Oncol. 2004;22:4958-65.

13. Pinder S, Provezano E, Earl H, Ellis IO. Laboratory handling and histology reporting of breast specimens from patients who have received neodjuvant chemotherapy. Histopathology. 2007;50:409-17.

14. Fan F. Evaluation and reporting of breast cancer after neoadjuvant chemotherapy. Open Pathol J. 2009;3:58-63.

15. Sahoo S, Lester S. Pathology of breast carcinomas after neoadjuvant chemotherapy: an overview with recommendations on specimen reporting and processing. Arch Pathol Lab Med. 2009;133:633-42.

16. Marchio S, Sapino A. The pathologic complete response open question in primary therapy. J Natl Cancer Inst Monogr. 2011;2011:86-90.

17. Provezano E, Bossuyt V, Viale G, Cameron D, Badve S, Denkert C, on behalf of the Residual Disease Characterization Working Group of the Breast International Group-North American Breast Cancer Group (BIG-NABCG) collaboration, et al. Standardization of pathological evaluation and reporting of postneoadjuvant specimens in clinical trials of breast cancer: recommendations from an international working group. Mod Pathol. 2015;28:1185-201.

18. Bossuyt V, Provenzano E, Summans W, Boughey J, Coles C, Curigliano G, the Breast International Group-North American Breast Cancer Group (BIGNABCG) collaboration, et al. Recommendations for standardized pathological of residual disease for neoadjuvant clinical trials of breast cancer by the BIGNABCG collaboration. Ann Oncol. 2015;26(7):1280-91.

19. Von Minckwitz G, Untch M, Blohmer J, Costa S, Eidtmann H, Fasching P, et al. Definition and impact of pathological complete response on prognosis after neoadjuvant chemotherapy in various intrinsic breast cancer subtypes. J Clin Oncol. 2012;30:1796-804.

20. Leong A, Zhuang $\mathrm{Z}$. The changing role of pathology in breast cancer diagnosis and treatment. Pathobiology. 2011;78(2):99-114.

21. Fitch K, Bernstein S, Aguilar M, Burnand B, LaCalle J, Lázaro P, et al. The RAND/UCLA appropriateness method user's manual/RAND. Available from: http://www.rand.org/pubsmonograph_reports/MR1269.html. Accessed 16 Jan 2017.

22. Slade S, Dionne C, Underwood M, Buchbinder R. Standardized method for reporting exercise programmes: protocol for a modified Delphi study. BMJ Open. 2014;4:e006682. doi:10.1136/bmjopen-2014-006682.

23. Diamond I, Grant R, Feldman B, Pencharz P, Ling S, Moore A, et al. Defining consensus: a systematic review recommends methodologic criteria for reporting of Delphi studies. J Clin Epidimiol. 2014;67:401-9.

24. Symmans W, Peintinger F, Hatzis C, Rajan R, Kuerer H, Valero V, et al Measurement of residual breast cancer burden to predict survival after neoadjuvant chemotherapy. J Clin Oncol. 2007;25:4414-22.

25. Edge S, Byrd D, Compton C, Fritz A, Greene F, Trotti A, American Joint Committee on Cancer (AJCC). Cancer staging manual. 7th ed. New York: Springer; 2009.

26. Residual cancer burden calculator and associated documents. Guide for measuring cancer cellularity, examples of gross and microscopic evaluation, pathology protocol for macroscopic and microscopic assessment of RCB. MD Anderson Cancer Center, Houston

27. Ogston K, Miller I, Payne S, Hutcheon A, Sarkar T, Smith I, et al. New histologic grade system to assess response of breast cancers to primary chemotherapy: prognostic significance and survival. Breast. 2003;12:320-7.

28. Rastogi P, Anderson S, Bear H, Geyer C, Kahlenberg M, Robidoux A et al. Preoperative chemotherapy: updates of national surgical adjuvant breast and bowel project protocols B-18 and B-27. J Clin Oncol. 2008;26(5):778-85.

29. Williams M, Eatrides J, Kim J, Talwar H, Esposito N, Szabunio M, et al. Comparison of breast magnetic resonance imaging clinical tumor size with pathologic tumor size in patients status post-neoadjuvant chemotherapy. Am J Surg. 2013;206(4):567-73. 
30. Marinovich M, Houssami N, Macaskill P, Sardanelli F, Irwig L, Mamounas E, et al. Meta-analysis of magnetic resonance imaging in detecting residual breast cancer after neoadjuvant therapy. J Natl Cancer Inst. 2013;105(5):321-33.

31. Rajan R, Esteva F, Symmans W. Pathologic changes in breast cancer following neoadjuvant chemotherapy: implications for the assessment of response. Clin Breast Cancer. 2004;5:235-8.

32. Marchio C, Maletta F, Annaratone L, Sapino A. The perfect pathology report after neoadjuvant therapy. J Natl Cancer Inst Monogr. 2015;51:47-50.

33. Hammond M, Hayes D, DowsettM Allred D, Hagerty K, Badve S, et al. American Society of Clinical Oncology/College of American pathologists guideline recommendations for immunohistochemical testing of estrogen and progesterone receptors in breast cancer (unabridged version). Arch Pathol Lab Med. 2010;134(7):48-72.

34. Harris L, Ismaila N, McShane L, Andre F, Collyar D, Gonzalez_angulo A, et al. Use of biomarkers to guide decisions on adjuvant systemic therapy for women with early-stage invasive breast cancer: American Society of Clinical Oncology clinical practice guideline. J Clin Oncol. 2016;34(10):1134-50.

35. Loi S, Symmans W, Bartlett J, Fumagalli D, Van't Veer L, Forbes J, et al. Proposals for uniform collection of biospecimens from neoadjuvant breast cancer clinical trials: timing and specimen types. Lancet Oncol. 2011;12:1162-8.

36. Braeuning M, Burke E, Pisano E. Embolization coils as tumors markers for mammography in patients undergoing neoadjuvant chemotherapy for carcinoma of the breast. Am J Roentgenol. 2000;174:251-2.

37. Houssami N, Ciatto S, Turner R, Cody H 3rd, Macaskill P. Preoperative ultrasound-guided needle biopsy of axillary nodes in invasive breast cancer: meta-analysis of its accuracy and utility in staging the axilla. Ann Surg. 2011;254:243-51.

38. Kuehn T, Bauerfeind I, Fehm T, Fleige B, Hausschild M, Helms G, et al. Sentinel-lymph-node biopsy in patients with breast cancer before and after neoadjuvant chemotherapy (SENTINA): a prospective multicentre cohort study. Lancet Oncol. 2013;14:609-6018.

39. Sataloff D, Mason B, Prestipino A, Seinige U, Lieber C, Baloch Z. Pathologic response to induction chemotherapy in locally advanced carcinoma of the breast: a determinant of outcome. J Am Coll Surg. 1995;180:297-306.

40. Sinn H, Schmid H, Junkermann H, Huober J, Leppien G, Kaufmann M, et al. Histologic regression of breast cancer after primary (neoadjuvant) chemotherapy. Geburtshilfe und Fraunheilkunde. 1994;54:552-8.

41. Peintinger F, Sinn B, Hatzis C, Albarracin C, Downs-Kelly E, Morkowski J, et al. Reproducibility of residual cancer burden for prognostic assessment of breast cancer after neoadjuvant chemotherapy. Mod Pathol. 2015;28(7):913-20.
42. Hennessy B, Hortobagyi G, Rouzier R, Kuerer H, Sneige N, Buzdar A, et al. Outcome after pathologic complete eradication of cytologically proven breas cancer axillary node metastases following primary chemotherapy. J Clin Oncol. 2005;23:9304-11.

43. Klauber-DeMore N, Ollila D, Moore D, Livasy C, Calvo B, Kim H, et al. Size of residual lymph node metastasis after neoadjuvant chemotherapy in locally advanced breast cancer patients is prognostic. Ann Surg Oncol. 2006;13:685-91.

44. Corben A, Abi-Raad R, Popa I, Teo C, Macklin E, Koerner F, et al. Pathologic response and long-term follow-up in breast cancer patients treated with neoadjuvant chemotherapy: a comparison between classifications and their practical applications. Arch Pathol Lab Med. 2013;137:1074-82.

45. Boughey J, Suman V, Mittendorf E, Ahrendt G, Wilke L, Taback B, et al. Sentinel lymph node surgery after neoadjuvant chemotherapy in patients with node-positive breast cancer. The ACOSOG Z1071 (Alliance) clinical trial. JAMA. 2013;310:1455-61.

46. Zhang N, Moran M, Huo Q, Haffty B, Yang Q. The hormonal receptor status in breast cancer can be altered by neoadjuvant chemotherapy: a meta-analysis. Caner Invest. 2011;29:594-8.

47. Von Minckwitz G, Schmitt W, Loibl S, Müller B, Blohmer J, Sinn B, et al. Ki67 measured after neoadjuvant chemotherapy for primary breast cancer. Clin Cancer Res. 2013;19:4521-31.

48. Polley M, Leung S, McShane L, Gao D, Hugh J, Mastropasqua M, et al. An international Ki67 reproducibility study. J Natl Cancer Inst. 2013;105:1897-906.

49. Sheri A, Smith I, Johnston S, A'Hern R, Nerurkar A, Jones R, et al. Residual proliferative cancer burden to predict long-term outcome following neoadjuvant chemotherapy. Ann Oncol. 2015;26:75-80.

50. US Food and Drug Administration. Guidance for industry: pathological complete response in neoadjuvant treatment of high-risk early-stage breast cancer: use as an endpoint to support accelerated approval (2014). http://www.fda.gov/ down;oads/Drugs/GuidanceComplianceRegulatoryInformation/Gui. Accessed 26 Feb 2017.

51. Mazouni C, Peintinger F, Wan-Kau S, Andre F, Gonzalo-Angulo A, Symmans $\mathrm{W}$, et al. Residual ductal carcinoma in situ in patients with complete eradication of invasive breast cancer after neoadjuvant chemotherapy does not adversely affect patient outcome. J Clin Oncol. 2007;25:2650-5. 